TRANSACTIONS OF THE

AMERICAN MATHEMATICAL SOCIETY

Volume 350, Number 1, January 1998, Pages 331-345

S 0002-9947(98)01778-4

\title{
SUR LA MULTIPLICITÉ DE LA PREMIÈRE VALEUR PROPRE DE L'OPÉRATEUR DE SCHRÖDINGER AVEC CHAMP MAGNÉTIQUE SUR LA SPHÈRE $S^{2}$
}

\author{
GÉRARD BESSON, BRUNO COLBOIS, AND GILLES COURTOIS
}

\begin{abstract}
RÉsumé. L'objet de cet article est d'étudier la multiplicité de la première valeur propre de l'opérateur de Schrödinger avec champ magnétique sur la sphère $S^{2}$, et, répondant en cela à une question posée par Y. Colin de Verdière, de montrer d'une part que cette multiplicité peut être arbitrairement grande, mais que, d'autre part, elle est toujours bornée en fonction de la courbure de la connexion associée.

Abstract. The purpose of this text is to study the first eigenvalue of the Schrödinger operator with magnetic field on the 2-sphere and to show that its multiplicity can be arbitrarily high. We also show that this multiplicity is bounded in terms of the curvature of the corresponding connection. This answers a question asked by Y. Colin de Verdière.
\end{abstract}

\section{INTRODUCTION}

L'opérateur de Schrödinger avec champ magnétique $H_{B, g}$ sur une variété riemannienne $(X, g)$ est le laplacien associé à une connexion hermitienne d'un fibré en droites complexes $\Pi: E \longrightarrow X$ dont la courbure est la 2-forme différentielle $B$ sur $X$. On dit que l'opérateur de Schrödinger est à champ magnétique et électrique si, à $H_{B, g}$, on ajoute un potentiel $V \in C^{\infty}(X, \mathbb{R})$. Dans la suite, on considère comme variété de base $(X, g)$ la sphère $S^{2}$ munie de sa métrique standard. De la thèse de Nabila Torki, [TI], on peut déduire [C-T]

Théorème [C-T]. Pour tout entier $N$, il existe un opérateur de Schrödinger avec champ magnétique et électrique sur le fibré trivial à $S^{2}$ ayant sa première valeur propre $\lambda_{1}$ de multiplicité $N$.

L'idée de N. Torki consiste à utiliser les fibrés canoniques de degré $N$ que l'on peut construire sur la sphère $S^{2}$ et dont on montre par un calcul explicite que la multiplicité de la première valeur propre pour la connexion canonique (i.e. avec un champ magnétique constant) est $N+1$ ([TI], Ch.II, §4). Puis, après avoir ôté un petit disque à la sphère, on trivialise le fibré et on ajoute un grand potentiel $V$ dans le disque rebouché. Les techniques de perturbation et de stabilité de Y. Colin de Verdière permettent alors d'assurer une grande multiplicité.

Une question naturelle est donc de savoir si la présence d'un champ électrique est nécessaire pour obtenir une multiplicité arbitrairement grande sur un fibré en droites complexes de $S^{2}$ (voir [CV2], §II, B(b)). Une autre question naturelle est

Received by the editors May 1, 1995 and, in revised form, April 2, 1996.

1991 Mathematics Subject Classification. Primary 58G25, 35P15, 53C21.

(C) 1998 American Mathematical Society 
de savoir s'il y a des obstructions "géométriques" à avoir $\lambda_{1}$ de grande multiplicité, la géométrie du fibré étant contenue dans la courbure de la connexion.

Dans cet article, nous construisons des exemples d'opérateur de Schrödinger avec champ magnétique sur $S^{2}$ avec première valeur propre $\lambda_{1}$ de grande multiplicité.

Théorème 1. Pour tout fibré en droites complexes sur $S^{2}$ et pour tout entier $N$, il existe un opérateur de Schrödinger avec champ magnétique ayant sa première valeur propre de multiplicité $N$.

Remarque. Nous décrivons les détails de la construction dans le cas du fibré trivial, l'extension aux fibrés quelconques consistent à s'y ramener (voir 5.2).

Les exemples du théorème 1 ont une courbure tendant vers l'infini avec $N$. Nous montrons que cela est nécessaire. Par exemple la $L^{2}$-norme de la courbure donne une borne de la multiplicité de $\lambda_{1}$.

Théorème 2. Il existe une constante $C$, ne dépendant pas du choix de la connexion, telle que la multiplicité de la première valeur propre de $H_{B}$ est bornée par $C\left(1+\|B\|_{L^{2}}^{2}\right)$.

Remarque. Les résultats de N. Torki ([TI]) montrent que pour une connexion à courbure constante sur un fibré de degré $m$, la première valeur propre à une multiplicité qui est $(m+1)$. La borne ci-dessus n'est pas optimale lorsque $m$ est grand et lorsque le champ magnétique est constant. Néanmoins, remarquons que la courbure ayant une intégrale fixe, la norme $L^{2}$ de celle-ci contrôle (en partie) ses variations. Le théorème 2 exprime que si celles-ci ne sont pas trop grandes alors la multiplicité est bornée ; le théorème 1, en revanche, montre que l'on peut forcer la multiplicité dans le cas contraire.

Au paragraphe 2 , on va démontrer le théorème 2. Puis, dès le paragraphe 3 , on construira un exemple permettant de démontrer le théorème 1. L'idée est de construire sur $S^{2}$ une connexion radiale et de décomposer l'opérateur de Schrödinger associé $H$ en une somme

$$
H=\bigoplus_{n \in \mathbb{Z}} H_{n}
$$

d'opérateurs de Schrödinger invariants par l'action isométrique naturelle de $S^{1}$ sur $S^{2}$, vue comme surface de rotation. Il est alors possible de prescrire la première valeur propre de $H_{1}, \ldots, H_{N}$ et d'obtenir ainsi une grande multiplicité.

\section{PREUVE DU ThÉORÈME 2}

On commence par rappeler la notion de connexion sur un fibré hermitien en droites complexes. Les détails se trouvent, par exemple, au chapitre 2 de [TI].

Soit $\mathbb{C} \hookrightarrow E \stackrel{\Pi}{\longrightarrow} S^{2}$ un fibré hermitien de rang 1 sur $S^{2}$. Soit $\nabla$ une connexion hermitienne. Dans une trivialisation $\Pi^{-1}(U) \cong U \times \mathbb{C}$, on a : $\nabla s=d s+i \alpha s$, où $s$ est une section du fibré, $\alpha$ une 1-forme réelle sur $U$. La courbure $B$ du fibré est une 2-forme sur $S^{2}$, définie localement par $B=i d \alpha$.

Rappelons ([KO]) que $\frac{1}{2 \pi i} B$ est entière sur $S^{2}$ (i.e. $\frac{1}{2 \pi i} \int_{S^{2}} B \in \mathbb{Z}$ ), et que, pour toute 2 -forme $B$ sur $S^{2}$ avec $\frac{1}{2 \pi i} B$ entière, il existe un fibré en droites complexes sur $S^{2}$ et une connexion $\nabla$ de courbure $B$. On dira que le fibré est de degré $m$ si $\frac{1}{2 \pi i} \int_{S^{2}} B=m$. 
Définition 2.1. L'opérateur de Schrödinger $H_{B}$ associé à une connexion $\nabla$ de courbure $B$ est l'application $H_{B}: C^{\infty}\left(S^{2}, E\right) \longrightarrow C^{\infty}\left(S^{2}, E\right)$ définie par :

$$
\int_{S^{2}}\left\langle H_{B} s, s\right\rangle=\int_{S^{2}}|\nabla s|^{2}=q(s)
$$

$s$ étant une section du fibré $E$ et $\langle, \quad\rangle$ désignant la structure hermitienne.

2.2. Expression de $H_{B}$. Si $D$ est la connexion de Levi-Civita de $S^{2}$ et $\left(e_{1}, e_{2}\right)$ un repère orthonormé local sur $S^{2}$, on a

$$
H_{B} s=-\sum_{i=1}^{2}\left(\nabla_{e_{i}} \nabla_{e_{i}} s-\nabla_{D_{e_{i}} e_{i}} s\right) .
$$

Proposition 2.4. Soit $\lambda_{1}$ la première valeur propre de $H_{B}$. Alors :

$$
\lambda_{1} \leq \frac{3}{2 \pi}\|B\|^{2}
$$

où $\|B\|$ désigne la $L^{2}$-norme de $B$ sur $S^{2}$.

Preuve du théorème 2. Une borne sur la courbure de la connexion implique donc une borne pour $\lambda_{1}$. Or, il découle de l'inégalité de Kato (Voir [BE], th. 6, p. 389 et la remarque au bas de la page 390.) que le nombre $N(\lambda)$ de valeurs propres de $H_{B}$ inférieures à $\lambda$ est borné en fonction de $\lambda$. Plus précisément, $N(\lambda) \leq C(1+\lambda)$, où $C$ est une constante qui se calcule explicitement en fonction d'un minorant de la courbure de Ricci de la variété de base (ici $S^{2}$ avec sa métrique standard) et d'un majorant de son diamètre ([GA], p. 366).

En conséquence :

$$
\text { mult } \lambda_{1}=N\left(\lambda_{1}\right) \leq C\left(1+\lambda_{1}\right) \leq \frac{3}{2 \pi} C\left(1+\|B\|^{2}\right) .
$$

La multiplicité de $\lambda_{1}$ est donc bornée en fonction de la valeur de $\lambda_{1}$ et donc en fonction de $\|B\|^{2}$, par la proposition 2.4.

Preuve de la proposition 2.4. Notons $\Omega_{0}$ la forme volume de $S^{2}$. Si $\frac{1}{2 \pi i} B$ est entière, d'intégrale égale à $m \in \mathbb{Z}$, on a, par la décomposition de Hodge,

$$
\frac{1}{2 \pi i} B=\frac{m}{4 \pi} \Omega_{0}+d \gamma \Rightarrow B=\frac{m}{2} i \Omega_{0}+2 \pi i d \gamma
$$

où $\gamma$ est une 1-forme réelle, cofermée. Il suffit, pour trouver une majoration de $\lambda_{1}$, d'exhiber une section $s$ de $E$ avec

$$
\frac{q(s)}{\|s\|^{2}} \leq \frac{3}{2 \pi}\|B\|^{2} .
$$

Si $P$ est un point de $S^{2}$ et $B(\varepsilon)$ est la boule de $S^{2}$ centrée en $P$ et de rayon $\varepsilon>0$, le fibré se trivialise sur $S^{2} \backslash B(\varepsilon)$ et $B(2 \varepsilon)$. Chaque section $s$ donne lieu à deux fonctions $s_{1}: S^{2} \backslash B(\varepsilon) \rightarrow \mathbb{C}$ et $s_{2}: B(2 \varepsilon) \rightarrow \mathbb{C}$, telles que sur $\left(S^{2} \backslash B(\varepsilon)\right) \cap B(2 \varepsilon)$ on a

$$
s_{2}(x)=\xi(x) s_{1}(x)
$$

où $\xi:\left(S^{2} \backslash B(\varepsilon)\right) \cap B(2 \varepsilon) \rightarrow S^{1} \subset \mathbb{C}$ est de degré $m$.

Nous choisissons la section définie en coordonnées polaires par :

$$
s_{1}(x)= \begin{cases}\sin (r)-\sin (2 \varepsilon) & \text { sur } S^{2} \backslash B(2 \varepsilon), \\ 0 & \text { sur } B(2 \varepsilon) \backslash B(\varepsilon),\end{cases}
$$


et

$$
s_{2}(x) \equiv 0 \text { sur } B(2 \varepsilon) .
$$

Il est clair que si l'on fait tendre $\varepsilon$ vers 0 , les calculs sont les mêmes que si l'on travaille avec $s(x)=\sin (r)$ sur $S^{2} \backslash\{P\}$. Nous ferons par la suite cet abus de langage. On a donc :

$$
\|s\|^{2}=\int_{0}^{\pi} d r \int_{0}^{2 \pi} d \theta \sin ^{2} r \sin r
$$

avec $\Omega_{0}=\sin r d r \wedge d \theta$.

Ainsi :

$$
\|s\|^{2}=\frac{8 \pi}{3} .
$$

Pour estimer $\|\nabla s\|^{2}$, on écrit la forme de connexion en coordonnées comme suit : $\alpha=2 \pi \gamma+\frac{m}{2}(1-\cos r) d \theta$.

En effet : $B=i d \alpha=2 i \pi d \gamma+\frac{m}{2} i \Omega_{0}$.

De (1) on déduit

$$
\|d \gamma\|^{2}+m^{2} \frac{1}{4 \pi}=\frac{1}{4 \pi^{2}}\|B\|^{2} .
$$

Enfin, on a par le minimax

$$
\|d \gamma\|^{2} \geq 2\|\gamma\|^{2}
$$

(2 est la première valeur propre non nulle de la sphère $S^{2}$ ). On a, puisque $s$ est réelle,

$$
\begin{aligned}
\nabla s & =d s+i\left(2 \pi \gamma s+\frac{m}{2}(1-\cos r) s d \theta\right), \\
|\nabla s|^{2} & =|d s|^{2}+\left|2 \pi \gamma s+\frac{m}{2}(1-\cos r) s d \theta\right|^{2}, \\
\int_{0}^{\pi} \int_{0}^{2 \pi}|d s|^{2} \sin r d r d \theta & =2 \pi \int_{0}^{\pi} \sin r \cos ^{2} r d r=\frac{4 \pi}{3}, \\
\int_{S^{2}}\left|2 \pi \gamma s-\frac{m}{2}(1-\cos r) s d \theta\right|^{2} & \leq \int_{S^{2}} 2\left(4 \pi^{2}|\gamma|^{2} \sin ^{2} r+\frac{m^{2}}{4}(1-\cos r)^{2}\right) .
\end{aligned}
$$

On a, par (3) :

$$
\int_{S^{2}}|\gamma|^{2} \leq \frac{1}{2} \int_{S^{2}}|d \gamma|^{2}
$$

et donc, par (2) :

$$
\begin{gathered}
\int_{S^{2}}|\gamma|^{2} \leq \frac{1}{2}\left(\frac{1}{4 \pi^{2}}\|B\|^{2}-\frac{1}{4 \pi} m^{2}\right), \\
\|\nabla s\|^{2} \leq \frac{4 \pi}{3}+\int_{S^{2}} 8 \pi^{2}|\gamma|^{2}+\frac{m^{2}}{2} 2 \pi \int_{0}^{\pi}(1-\cos r)^{2} \sin r d r \\
\leq \frac{4 \pi}{3}+\frac{8 \pi m^{2}}{3}+\|B\|^{2}-m^{2} \pi=\frac{4 \pi}{3}+\frac{5 \pi}{3} m^{2}+\|B\|^{2} .
\end{gathered}
$$

En outre :

$$
\left|\int_{S^{2}} B\right| \leq\left(\int_{S^{2}}|B|^{2}\right)^{\frac{1}{2}} \sqrt{4 \pi} \Rightarrow 4 \pi^{2} m^{2} \leq 4 \pi\|B\|^{2} \Rightarrow\|B\|^{2} \geq \pi m^{2} .
$$


Si $m \neq 0:\|\nabla s\|^{2} \leq \frac{4}{3}\left(\frac{1}{m^{2}+1}\right)\|B\|^{2} \leq 4\|B\|^{2}$

Si $m=0$ : on choisit $s \equiv 1$ au lieu de $s=\sin r$. On a :

$$
\|\nabla s\|^{2} \leq 4 \pi^{2} \int_{S^{2}}|\gamma|^{2} \leq 2 \pi^{2} \frac{1}{4 \pi^{2}}\|B\|^{2} \Rightarrow \frac{\|\nabla s\|^{2}}{\|s\|^{2}} \leq \frac{\|B\|^{2}}{8 \pi} .
$$

\section{Construction D'une Connexion}

On aborde ici la construction d'une famille de connexions sur le fibré trivial $S^{2} \times \mathbb{C}$ permettant de montrer l'existence d'opérateurs de Schrödinger dont la première valeur propre est de multiplicité arbitrairement grande. Sur $S^{2}$, on écrit la métrique en coordonnées polaires géodésiques $g(r)=d r^{2}+\sin ^{2} r d \theta, 0<r<\pi, 0<\theta<2 \pi$.

On cherche des connexions du type $\nabla=d+i \alpha$, où $\alpha$ est une 1-forme, réelle, cofermée, ne dépendant que de $r$. Soit $f: S^{2} \longrightarrow \mathbb{R}$, s'écrivant $f=f(r)$ en coordonnées polaires. À $f$ est associée la 2-forme différentielle $* f=f \sin r d r \wedge d \theta$ où * est l'opérateur de Hodge. On considère $\alpha=\delta * f=f^{\prime}(r) \sin r d \theta, \delta$ codifférentielle. Un calcul élémentaire montre que :

Proposition 3.1. En coordonnées polaires, l'opérateur de Schrödinger $H_{f}$ associé à $f$ s'écrit :

$$
H_{f} s=-\frac{\partial^{2} s}{\partial r^{2}}-\frac{1}{\sin ^{2} r} \frac{\partial^{2} s}{\partial \theta^{2}}-\cot r \frac{\partial s}{\partial r}-\frac{2 i}{\sin r} f^{\prime}(r) \frac{\partial s}{\partial \theta}+f^{\prime 2}(r) s
$$

Remarque 3.2 .

$$
\Delta s=-\frac{\partial^{2} s}{\partial r^{2}}-\frac{1}{\sin ^{2} r} \frac{\partial^{2} s}{\partial \theta^{2}}-\cot r \frac{\partial s}{\partial r}
$$

est le laplacien usuel $\Delta_{S^{2}}$ sur $S^{2}$.

Il est alors possible de décomposer $s$ en série de Fourier comme suit :

$$
s(r, \theta)=\sum_{n=-\infty}^{\infty} a_{n}(r) e^{i n \theta} .
$$

L'opérateur de Schrödinger $H_{f}$ commute avec cette décomposition. On obtient alors :

\section{Proposition 3.3.}

$$
H_{f} s=\sum_{n=-\infty}^{\infty} e^{i n \theta}\left(\Delta_{S^{2}} a_{n}+\left(\frac{n}{\sin r}+f^{\prime}(r)\right)^{2} a_{n}\right) .
$$

Définition 3.4. On écrit

$$
H_{f} s=\sum_{n=-\infty}^{\infty} e^{i n \theta} H_{f}^{n}\left(a_{n}\right)
$$

où l'on a posé $H_{f}^{n}(a)=\Delta_{S^{2}} a+\left(\frac{n}{\sin r}+f^{\prime}\right)^{2} a$.

L'étude du spectre de $H_{f}$ se ramène à l'étude des spectres des opérateurs $H_{f}^{n}$ ainsi définis.

Remarque 3.5. Les opérateurs $H_{f}^{n}$ sont réels et 1-dimensionnels, et sont définis dans $L^{2}([0, \pi], \sin r d r)$. Si $s$ est une section propre $H_{f} s=\lambda s$ et $s=\sum_{n=-\infty}^{\infty} a_{n}(r) e^{i n \theta}$, alors $H_{n}^{f}\left(a_{n}\right)=\lambda a_{n}$ pour tout $n \in \mathbb{N}$. (Seul un nombre fini de $n$ sont tels que $a_{n} \not \equiv 0$ ). 
Si $n \neq 0$, le domaine de la forme quadratique $q_{n}$ associée à $H_{n}^{f}$ est le complété dans $L^{2}([0, \pi], \sin r d r)$ pour la norme définie par $q_{n}$ de l'espace des fonctions $C^{1}(] 0, \pi[)$ s'annulant en 0 et $\pi$; si $n=0$, la condition aux limites est alors $u^{\prime}(0)=u^{\prime}(\pi)=0$.

Pour ces conditions au bord, les opérateurs $H_{n}^{f}$ sont auto-adjoints, à résolvante compacte. Leur spectre noté $\left\{\lambda_{i, n}^{f}\right\}_{i \in \mathbb{N}}$ est discret. Si $\left\{\lambda_{i}^{f}\right\}_{i \in \mathbb{N}}$ est le spectre de $H_{f}$, on a :

$$
\left\{\lambda_{i}^{f}\right\}_{i \in \mathbb{N}}=\bigcup_{n \in \mathbb{Z}}\left\{\lambda_{i, n}^{f}\right\}_{i \in \mathbb{N}}
$$

\section{Construction du Champ magnétique}

Dans ce paragraphe, nous construisons une famille de connexions du type précédent (radiales) qui donnera lieu à une première valeur propre $\lambda_{1}^{f}$ de multiplicité $N$, entier arbitrairement fixé, pour $H^{f}$. La situation idéale consisterait à partager la sphère en bandes radiales $J_{1}, \ldots, J_{n}$, elles-mêmes séparées par des intervalles (Figure 1), et à pouvoir choisir $f$ de sorte que $H_{n}^{f}(1 \leq n \leq N)$ admette un potentiel infini entre les $J_{k}$, et un potentiel petit, parfois nul, sur les $J_{k}$. Dans ce cas, on se ramènerait au problème de Dirichlet sur les intervalles $J_{k}$, puisqu'à cause du potentiel infini, les fonctions propres de $H_{n}^{f}$ devraient être nulles hors des $J_{k}$. On pourrait alors ajuster la longueur des $J_{k}$ de sorte que $\lambda_{1,1}^{f}=\lambda_{1,2}^{f}=\cdots=\lambda_{1, N}^{f}$, ce qui permettrait de conclure (pour peu que $\lambda_{1, n}^{f}>\lambda_{1,1}^{f} \quad \forall n \notin\{1, \cdot, N\}$ ).

L'objet de ce paragraphe est d'imiter cette situation idéale. Plus précisément, nous construisons une famille de fonctions $f_{\varepsilon}$, de sorte que, lorsque $\varepsilon \rightarrow 0, \lambda_{1, k}^{f_{\varepsilon}}$ converge vers la première valeur propre de la bande $J_{k}$ (avec condition de Dirichlet) pour $1 \leq k \leq N$. On conclura ensuite par un argument de perturbation comme dans [CV1].

4.1. Choix des $J_{k}$. Dans la suite et puisque les objets considérés sont radiaux nous identifierons systématiquement les bandes parallèles sur la sphère aux intervalles de $[0, \pi]$ qui les définissent.

On décompose l'intervalle $[0, \pi]$ en une réunion

$$
[0, \pi]=\bigcup_{k=1}^{N}\left[x_{k-1}, x_{k}\right] \cup\left[0, \frac{1}{\sqrt{N}}\right] \cup\left[\pi-\frac{1}{\sqrt{N}}, \pi\right]
$$

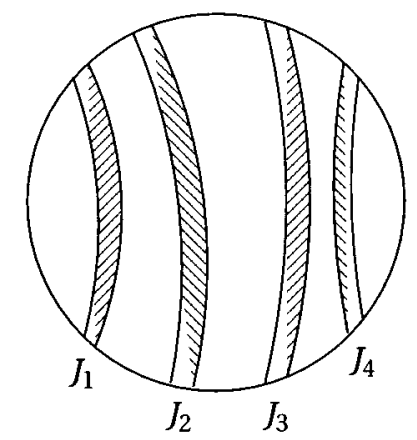

Figure 1 
où

$$
x_{k}=\pi\left(\frac{1}{\sqrt{N}}+\frac{1-2 / \sqrt{N}}{N} k\right), \quad k=0, \ldots, N,
$$

on pose $I_{k}=\left[x_{k-1}, x_{k}\right]$.

Rappelons que nous travaillons sur $[0, \pi]$ avec l'élément de volume sphérique, c'est-à-dire $\sin r d r$ (où $r$ est la coordonnée sur $[0, \pi]$ ) que nous comparerons avec la situation euclidienne, c'est-à-dire $d r$. C'est la raison pour laquelle nous nous plaçons à distance raisonnable (de l'ordre de $\frac{1}{\sqrt{N}}$ ) de 0 et $\pi$ où $\sin (r) d r$ dégénère.

Pour un intervalle $[a, b] \subset[0, \pi]$, notons $\mu([a, b])$ la première valeur propre, avec condition de Dirichlet en $a$ et $b$, de l'opérateur $\Delta_{S^{2}}$ restreint aux fonctions radiales, c'est-à-dire

$$
\Delta_{S^{2}} v=-v^{\prime \prime}(r)-(\operatorname{cotg}(r)) v^{\prime}(r) .
$$

C'est l'opérateur associé à la forme quadratique

$$
q(u)=\int_{0}^{\pi}\left|u^{\prime}(r)\right|^{2} \sin (r) d r
$$

$\operatorname{sur} L^{2}([0, \pi], \sin (r) d r)$.

Nous allons construire $\bar{J}_{k} \subset I_{k}$ pour $1 \leq k \leq N$ en sorte que $\mu\left(\bar{J}_{k}\right)=\mu_{0}$ où l'on définit comme suit :

Soit $k_{0}$ tel que $\mu\left(I_{k_{0}}\right)=\max \left\{\mu\left(I_{k}\right), k=1, \ldots, N\right\}$. Soit $\bar{y}_{k_{0}} \in I_{k_{0}}$ tel que $\frac{\bar{y}_{k_{0}}-x_{k_{0}-1}}{x_{k_{0}}-x_{k_{0}-1}}=\frac{9}{10}$, alors on pose $\mu_{0}=\mu\left(\left[x_{k_{0}-1}, \bar{y}_{k_{0}}\right]\right)$. Par la monotonie de $\mu$ on a

$$
\mu\left(I_{k}\right) \leq \mu\left(I_{k_{0}}\right) \leq \mu_{0}
$$

De même la fonction $\mu\left(\left[x_{k-1}, x\right]\right)$ étant croissante, pour chaque $k=1, \ldots, N$, il existe $\bar{y}_{k} \in I_{k}$ tel que $\mu\left(\left[x_{k-1}, \bar{y}_{k}\right]\right)=\mu_{0}$. On définit alors $\bar{J}_{k}=\left[x_{k-1}, \bar{y}_{k}\right]$. Enfin on pose $J_{k}=\left[x_{k-1}, y_{k}\right]$, où $y_{k}$ est proche de $\bar{y}_{k}$, précisément (voir Figure 2 )

$$
\left|y_{k}-\bar{y}_{k}\right| \leq e^{-N} \text {. }
$$

( $N$ sera choisi très grand pour que $\left|\mu\left(J_{k}\right)-\mu\left(\bar{J}_{k}\right)\right| \leq 1 / 4$.)

Nous allons montrer la convergence de $\lambda_{1, k}^{f_{\varepsilon}}$ vers la première valeur propre avec condition de Dirichlet de $\Delta_{S^{2}}$ sur $J_{k}$, uniformément par rapport à $y_{k}$, dans le voisinage choisi. Ce choix de l'intervalle $\left[y_{k}, x_{k}\right]$ bien plus petit que $\left[x_{k-1}, y_{k}\right]$ facilitera le contrôle du spectre des $H_{n}^{f_{\varepsilon}}$.

Les intervalles $\left[y_{k}, x_{k}\right](k=1, \ldots, N)$ seront ceux sur lesquels le potentiel sera très grand. On laisse une certaine "marge de liberté" à $y_{k}$, afin de pouvoir appliquer plus tard les méthodes de perturbations.

4.2. Construction de $f_{\varepsilon}$. Soit $\delta=\varepsilon / N^{2}$ et $0<\varepsilon<1$. On définit $f_{\varepsilon}$ comme suit :

$$
\begin{aligned}
& \text { Sur } J_{k}=\left[x_{k-1}, y_{k}\right] \quad f_{\varepsilon}^{\prime}=-\frac{k}{\sin r} \\
& \text { Sur }\left[y_{k}+\delta, x_{k}-\delta\right] \quad f_{\varepsilon}^{\prime}=e^{N / \varepsilon} \\
& \text { Sur }[0, \delta] \text { et }[\pi-\delta, \pi] \quad f_{\varepsilon}^{\prime}=0 \\
& \text { Sur }\left[2 \delta, x_{0}-\delta\right] \quad f_{\varepsilon}^{\prime}=e^{1 / \varepsilon r} \\
& \text { Sur }\left[y_{N}+\delta, \pi-2 \delta\right] \quad f_{\varepsilon}^{\prime}=e^{1 / \varepsilon(\pi-r)}
\end{aligned}
$$

Sur les autres intervalles on raccorde à l'aide de fonctions $\mathcal{C}^{\infty}$ et monotones.

Comme nous l'avions annoncé le potentiel pour l'opérateur $H_{k}^{f_{\varepsilon}}$, pour $1 \leq k \leq N$, tend vers l'infini en dehors de $J_{k}$. 


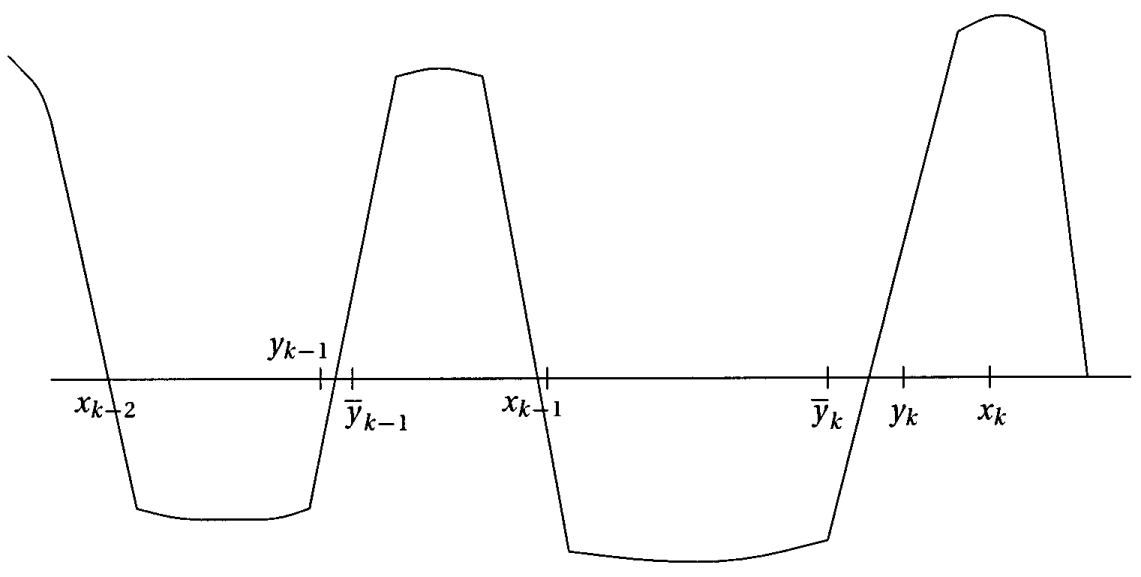

Figure 2

Dans la suite nous utiliserons $H_{k}^{\varepsilon}$ et $\lambda_{1, k}^{\varepsilon}$ au lieu de $H_{k}^{f_{\varepsilon}}$ et $\lambda_{1, k}^{f_{\varepsilon}}$.

Convergence de $\lambda_{1, k}^{\varepsilon}$ pour $1 \leq k \leq N$.

Théorème 4.3. Avec les notations qui précèdent, on a

$$
\lambda_{1, k}^{\varepsilon} \underset{\varepsilon \rightarrow 0}{\longrightarrow} \mu\left(J_{k}\right)
$$

uniformément en $y_{k}$ dans l'intervalle défini précédemment.

Preuve.

- Majoration.

On utilise le principe du min-max. La fonction du problème de Dirichlet sur $J_{k}$ sert de fonction test. Comme le potentiel de $H_{k}^{\varepsilon}$ est nul sur $J_{k}$ on a

$$
\lambda_{1, k}^{\varepsilon} \leq \mu\left(J_{k}\right)
$$

- Minoration de liminf $\lambda_{1, k}^{\varepsilon}$.

Elle résulte d'une série de lemmes techniques qui permettent de préciser comment le potentiel choisi approxime la situation idéale décrite plus haut. Le lemme 4.4 permet de prouver qu'une fonction propre pour $H_{k}^{\varepsilon}$ doit être petite au voisinage des extrémités de $J_{n}(1 \leq n \leq N)$, c'est-à-dire proche de satisfaire les conditions de Dirichlet sur $J_{n}$. Le lemme 4.10 permet alors de comparer précisément le quotient de Rayleigh de cette fonction restreinte à $J_{n}$ avec $\mu\left(J_{n}\right)$. $1)$.

Soit $u$ la première fonction propre normalisée de $H_{k}^{\varepsilon}$ (i.e. $\quad \int_{0}^{\pi} u^{2}(r) \sin r d r=$

Lemme 4.4. Soit $1 \leq n \leq N$, alors il existe $a \in\left[x_{n-1}-2 \delta, x_{n-1}-\delta\right]$ et $b \in$ $\left[y_{n}+\delta, y_{n}+2 \delta\right]$ tel que $|u(a)| \leq \varepsilon$ et $|u(b)| \leq \varepsilon$. 
Preuve. Soit $U_{\varepsilon}=\left\{r \in\left[x_{n-1}-2 \delta, x_{n-1}-\delta\right] \backslash|u(r)|>\varepsilon\right\}$, alors

$$
\begin{aligned}
\mu\left(J_{n}\right) & \geq \lambda_{1, n}^{\varepsilon} \geq \int_{U_{\varepsilon}}\left(\left|u^{\prime}(r)\right|^{2}+\left(f_{\varepsilon}^{\prime}(r)+\frac{n}{\sin r}\right)^{2} u^{2}(r)\right) \sin (r) d r \\
& \geq e^{\frac{2 N}{\varepsilon}} \int_{U_{\varepsilon}} u^{2}(r) \sin (r) d r \geq \varepsilon^{2} e^{\frac{2 N}{\varepsilon}} \int_{U_{\varepsilon}} \sin (r) d r \\
& \geq \varepsilon^{2} e^{\frac{2 N}{\varepsilon}} \sin (1 / \sqrt{N}) \ell\left(U_{\varepsilon}\right)
\end{aligned}
$$

ou $\ell\left(U_{\varepsilon}\right)$ est la mesure de Lebesgue de $U_{\varepsilon}$. Par ailleurs par continuité de $\mu$ et parce que $y_{n}-x_{n-1}$ est minoré, $\mu\left(J_{n}\right)$ est majoré. Donc il existe une constante $C$ telle que

$$
\ell\left(U_{\varepsilon}\right) \leq C e^{-N / \varepsilon} / \varepsilon^{2}
$$

et donc pour $\varepsilon$ assez petit $\ell\left(U_{\varepsilon}\right)<\delta=\frac{\varepsilon}{N^{2}}$. Il existe donc $a \in\left[x_{n-1}-2 \delta, x_{n-1}-\delta\right]$ tel que $a \notin U_{\varepsilon}$, c'est-à-dire $|u(a)| \leq \varepsilon$. On fait de même avec $b$. Signalons que $N$ qui sera choisi très grand est fixé.

De même le lemme suivant permettra de traiter les extrémités de l'intervalle $[0, \pi]$.

Lemme 4.5. Il existe deux points $\left.c_{0} \in\right] 2 \delta, 3 \delta\left[\right.$ et $\left.c_{N} \in\right] \pi-3 \delta, \pi-2 \delta[$ tels que $\left|u\left(c_{0}\right)\right| \leq \varepsilon$ et $\left|u\left(c_{N}\right)\right| \leq \varepsilon$.

La preuve est similaire à celle du lemme 4.4. Par ailleurs du lemme 4.4, on déduit immédiatement les lemmes suivant :

Lemme 4.6. Sur les intervalles $\left[x_{n}-2 \delta, x_{n}\right]$ et $\left[y_{n}, y_{n}+2 \delta\right]$ on a:

$$
|u(x)| \leq \varepsilon+\lambda_{1, k}^{\varepsilon}(\sqrt{N} 2 \delta)^{1 / 2}=O(\varepsilon)^{1 / 2} .
$$

Lemme 4.7. $\int_{x_{n-1}}^{y_{n}} u^{2}(r) \sin r d r=\int_{a_{n-1}}^{b_{n}} u^{2}(r) \sin r d r+O\left(\varepsilon^{2}\right)$.

Lemme 4.8. On $a: \mu\left(\left[a_{n-1}, b_{n}\right]\right)=\mu\left(J_{n}\right)(1+O(\varepsilon))$ pour $n=1, \ldots, N$.

Preuve du lemme 4.6. On a $u(x)-u\left(a_{n}\right)=\int_{a_{n}}^{x} u^{\prime}(r) d t$ et

$$
\begin{aligned}
\int_{a_{n}}^{x} u^{\prime}(r) d t & \leq\left(\int_{a_{n}}^{x}\left(u^{\prime}(r)\right)^{2} \sin t d t\right)^{1 / 2}\left(\int_{a_{n}}^{x}(\sin t)^{-1} d t\right) \\
& \leq \lambda_{1, k}^{\varepsilon}(2 \delta \sqrt{N})^{1 / 2} \int_{0}^{\pi} u^{2}(t) \sin t d t=\lambda_{1, k}^{\varepsilon}(2 \delta \sqrt{N})^{1 / 2}
\end{aligned}
$$

Le lemme 4.7 découle immédiatement du lemme 4.6.

Preuve du lemme 4.8. Pour $N$ fixé, les intervalles $\left[a_{n-1}, b_{n}\right]$ et $S_{n}$ sont quasiisométriques (pour la métrique radiale venant de la sphère) de rapport $1+O(\varepsilon)$.

Remarque. Rappelons que l'intervalle $J_{k}=\left[x_{k-1}, y_{k}\right]$, où $y_{k}$ est voisin de $\bar{y}_{k}$. Nous allons montrer que $\mu\left(J_{k}\right)$ est borné supérieurement indépendamment de $k$ et $y_{k}$. Pour cela nous allons utiliser le lemme suivant qui sera utile par ailleurs. Pour un intervalle $[a, b]$ on note $\mu_{E}([a, b])$ la première valeur propre de l'opérateur $-\frac{d r^{2}}{d r^{2}}$ (Laplacien euclidien) sur $[a, b]$ avec conditions de Dirichlet, on rappelle que $\mu_{E}([a, b])=\frac{\pi^{2}}{(b-a)^{2}}$. Alors on a 
Lemme 4.9. Soit $[a, b] \subset] \frac{\pi}{\sqrt{N}}, \pi\left(1-\frac{1}{\sqrt{N}}\right)[$ de longueur inférieure à $\pi / N$, alors il existe $N_{0}$ tel que pour tout $N \geq N_{0}$, on a

$$
\frac{1}{D} \leq \frac{\mu([a, b])}{\mu_{E}([a, b])} \leq D
$$

avec $D=1,001$.

Preuve. Par le principe du min-max

$$
\frac{s}{S} \mu_{E}([a, b]) \leq \mu([a, b]) \leq \frac{S}{s} \mu_{E}([a, b])
$$

où $S=\sup \{\sin (r) \backslash r \in[a, b]\}$ et $s=\inf \{\sin (r) \backslash r \in[a, b]\}$. Par le théorème des accroissements finis

$$
S \leq s+\frac{\pi}{N}
$$

et de plus $s \geq \sin \left(\frac{\pi}{\sqrt{N}}\right)$, d'où

$$
\frac{S}{s} \leq 1+\frac{\pi}{N}\left(\sin \left(\frac{\pi}{\sqrt{N}}\right)\right)^{-1},
$$

ce qui prouve le lemme pour $N$ assez grand.

En conséquence pour $N \geq N_{0}$

$$
\mu\left(J_{k}\right) \leq D \mu_{E}\left(J_{k}\right)=\frac{\pi^{2} D}{\left(y_{k}-x_{k-1}\right)}=\frac{\pi^{2} D}{\left(\bar{y}_{k}-x_{k-1}-e^{-N}\right)}
$$

qui est la borne annoncée car $N, x_{k-1}$ et $\bar{y}_{k}$ sont fixés (indépendant de $\varepsilon$ ).

Lemme 4.10. On considère l'intervalle $\left[x_{n-1}-2 \delta, y_{n}+2 \delta\right]$ et une fonction $u \in$ $\mathcal{C}^{\infty}([0, \pi])$ telle qu'il existe $a \in\left[x_{n-1}-2 \delta, x_{n-1}-\delta\right]$ et $b \in\left[y_{n}+\delta, y_{n}+2 \delta\right]$ avec $|u(a)| \leq \varepsilon$ et $|u(b)| \leq \varepsilon$. Alors

$$
\int_{a}^{b}\left(u^{\prime}(r)\right)^{2} \sin (r) d r \geq \mu([a, b])(1+O(\varepsilon)) \int_{a}^{b}(u(r))^{2} \sin (r) d r+O(\varepsilon)
$$

lorsque $\varepsilon \longrightarrow 0$.

Preuve. Posons $\bar{u}(r)=u(r)-\frac{u(a)-u(b)}{a-b}(r-a)-u(a)$, alors $\bar{u}(a)=\bar{u}(b)=0$. On a donc

$$
\int_{a}^{b}\left(\bar{u}^{\prime}(r)\right)^{2} \sin (r) d r \geq \mu([a, b]) \int_{a}^{b}\left(\bar{u}^{2}(r)\right)^{2} \sin (r) d r
$$

De plus

i) $\bar{u}^{\prime}=u^{\prime}-\frac{u(a)-u(b)}{b-a}$ donne

$$
\begin{aligned}
\left(u^{\prime}\right)^{2} & \geq\left(\bar{u}^{\prime}\right)^{2}-\frac{4 \varepsilon}{b-a}\left(\bar{u}^{\prime}\right)^{2}-\frac{4 \varepsilon}{b-a} \\
& =\left(\bar{u}^{\prime}\right)^{2}\left(1-\frac{4 \varepsilon}{b-a}\right)-\frac{4 \varepsilon}{b-a}=\left(\bar{u}^{\prime}\right)^{2}(1+O(\varepsilon))+O(\varepsilon)
\end{aligned}
$$

(Dans la première inégalité nous avons utilisé la relation bien connue $x y \leq x+x y^{2}$ ).

ii) $|\bar{u}(r)| \geq|u(r)|-3 \varepsilon$ donne

$$
\begin{aligned}
\left.|| \bar{u}(r)\right|^{2}-|u(r)|^{2} \mid & \leq 3 \varepsilon(|\bar{u}(r)|+|u(r)|) \\
& \leq 3 \varepsilon(3 \varepsilon+2|u(r)|)=O(\varepsilon)(u(r))^{2}+O(\varepsilon),
\end{aligned}
$$


ainsi

$$
(\bar{u}(r))^{2} \geq(u(r))^{2}(1+O(\varepsilon))+O(\varepsilon) .
$$

Alors de i) et ii), on déduit :

$$
\begin{aligned}
\int_{a}^{b}\left(u^{\prime}(r)\right)^{2} \sin (r) d r & \geq(1+O(\varepsilon)) \int_{a}^{b}\left(\bar{u}^{\prime}(r)\right)^{2} \sin (r) d r+O(\varepsilon) \\
& \geq(1+O(\varepsilon)) \mu([a, b]) \int_{a}^{b}(\bar{u}(r))^{2} \sin (r) d r+O(\varepsilon) \\
& \geq \mu([a, b])(1+O(\varepsilon)) \int_{a}^{b}(u(r))^{2} \sin (r) d r+O(\varepsilon) .
\end{aligned}
$$

(Dans la dernière inégalité nous utilisons le fait que $\mu([a, b]) \leq \mu\left(J_{n}\right)$ et donc est borné supérieurement.) Remarquons que l'inégalité est uniforme en $y_{k}$ proche de $\bar{y}_{k}$.

Lemme 4.11. On considère l'intervalle $[0,3 \delta]$ (resp. $[\pi-3 \delta, \pi]$ ) et une fonction $u \in \mathcal{C}^{\infty}([0, \pi])$ telle qu'il existe $c_{0} \in[0,3 \delta]$ (resp. $c_{N} \in[\pi-3 \delta, \pi]$ ) avec $\left|u\left(c_{0}\right)\right| \leq \varepsilon$ (resp. $\left.\left|u\left(c_{N}\right)\right| \leq \varepsilon\right)$, alors

$$
\begin{aligned}
\int_{0}^{c_{0}}\left(u^{\prime}(r)\right)^{2} \sin (r) d r & \geq \frac{O(1)}{\varepsilon^{2}}\left(\int_{0}^{c_{0}} u^{2}(r) \sin r d r\right)(1+O(\varepsilon))+O(\varepsilon) \\
\text { (resp. } \int_{c_{N}}^{\pi}\left(u^{\prime}(r)\right)^{2} \sin (r) d r & \left.\geq \frac{O(1)}{\varepsilon^{2}}\left(\int_{c_{N}}^{\pi} u^{2}(r) \sin (r) d r\right)(1+O(\varepsilon))+O(\varepsilon)\right) .
\end{aligned}
$$

Lemme 4.12. Pour tout point $r \in] 0, \pi\left[\right.$, et $1 \leq n \leq N$, on $a: \frac{n}{\sin r}+e^{1 / \varepsilon r} \geq e^{1 / \varepsilon \pi}$.

La preuve est immédiate.

Procédons maintenant à la preuve de la minoration annoncée dans le théorème 4.3. Soit $u$ une fonction propre de $H_{k}$ et $q_{k}$ la forme quadratique associée à cet opérateur, c'est-à-dire

$$
q_{k}(u)=\int_{0}^{\pi}\left[\left(u^{\prime}(r)\right)^{2}+\left(\frac{k}{\sin (r)}+f_{\varepsilon}^{\prime}\right)^{2}(u(r))^{2}\right] \sin (r) d r .
$$

Des lemmes précédents il résulte que si $a_{n-1}, b_{n}, c_{0}$ et $c_{N}$ sont les points de $\left[x_{n-1}-2 \delta, x_{n-1}-\delta\right]$ et $\left[y_{n}+\delta, y_{n}+2 \delta\right]$ exhibés au lemme 4.4 et au lemme 4.5 , alors

1) Sur $\left[a_{n-1}, b_{n}\right]$ on a

$$
\int_{a_{n-1}}^{b_{n}}\left(u^{\prime}(r)\right)^{2} \sin (r) d r \geq(1+O(\varepsilon)) \mu\left(\left[a_{n-1}, b_{n}\right]\right) \int_{a_{n-1}}^{b_{n}} u^{2}(r) \sin (r) d r+O(\varepsilon)
$$

(lemme 4.10).

2) Sur $\left[b_{n}, a_{n+1}\right]$ on a $\left(\frac{k}{\sin r}+f_{\varepsilon}^{\prime}\right)^{2} \geq e^{2 N / \varepsilon} \geq e^{2 / \varepsilon \pi} \quad$ (lemme 4.12)

3) Sur $\left[c_{0}, a_{0}\right] \cup\left[b_{N}, c_{N}\right]$ le lemme 4.11 donne $\left(\frac{k}{\sin (r)}+f_{\varepsilon}^{\prime}\right)^{2} \geq e^{\frac{2}{\varepsilon \pi}}$.

4) Sur $J_{n}$ pour $k \neq n$ on vérifie aisément l'inégalité

$$
\left|\frac{k}{\sin (r)}+f^{\prime}(r)\right| \geq 1
$$


En regroupant ces inégalités nous obtenons

$$
\begin{aligned}
q_{k}(u) \geq & \sum_{n=1}^{N} \mu\left(\left[a_{n-1}, b_{n}\right]\right)(1+O(\varepsilon)) \int_{a_{n-1}}^{b_{n}} u^{2}(r) \sin (r) d r \\
& +\frac{O(1)}{\varepsilon^{2}}\left(\int_{0}^{c_{0}} u^{2}(r) \sin (r) d r+\int_{c_{N}}^{\pi} u^{2}(r) \sin (r) d r\right)(1+O(\varepsilon)) \\
& +O(\varepsilon)+\sum_{n=1}^{N} e^{\frac{2}{\pi \varepsilon}} \int_{b_{n}}^{a_{n+1}} u^{2}(r) \sin (r) d r+e^{\frac{2}{\pi \varepsilon}} \int_{c_{0}}^{a_{0}} u^{2}(r) \sin (r) d r \\
& +e^{\frac{2}{\pi \varepsilon}} \int_{b_{N}}^{c_{N}} u^{2}(r) \sin (r) d r+\sum_{n \neq k} \int_{x_{n-1}}^{y_{n}} u^{2}(r) \sin (r) d r .
\end{aligned}
$$

En utilisant les lemmes 4.7 et 4.8 , on obtient alors :

$$
\begin{aligned}
q_{k}(u) \geq & \mu\left(J_{k}\right)(1+O(\varepsilon)) \int_{J_{k}} u^{2}(r) \sin (r) d r \\
& +\sum_{n \neq k}\left(1+\mu\left(J_{n}\right)\right)(1+O(\varepsilon)) \int_{J_{n}} u^{2}(r) \sin (r) d r \\
& +\frac{O(1)}{\varepsilon^{2}}(1+O(\varepsilon))\left(\int_{0}^{c_{0}} u^{2}(r) \sin (r) d r+\int_{c_{N}}^{\pi} u^{2}(r) \sin (r) d r\right)+O(\varepsilon) \\
& +\sum_{n=1}^{N} e^{\frac{2}{\pi \varepsilon}} \int_{b_{n}}^{a_{n+1}} u^{2}(r) \sin (r) d r+e^{\frac{2}{\pi \varepsilon}} \int_{c_{0}}^{a_{0}} u^{2}(r) \sin (r) d r \\
& +e^{\frac{2}{\pi \varepsilon}} \int_{b_{N}}^{c_{N}} u^{2}(r) \sin (r) d r .
\end{aligned}
$$

Rappelons que les intervalles $\bar{J}_{n}$ sont choisis en sorte que $\mu\left(\bar{J}_{n}\right)=\mu_{0}$ pour tout $n$. Les intervalles $J_{n}$ sont des petites perturbations des $\bar{J}_{n}$, en particulier on a choisi $N$ (qui est fixé) assez grand pour que $\left|\mu\left(J_{n}\right)-\mu\left(\bar{J}_{n}\right)\right| \leq 1 / 4$, ce qui implique l'inégalité

$$
1+\mu\left(J_{n}\right) \geq 3 / 4+\mu_{0} \geq 1 / 2+\mu\left(J_{k}\right) .
$$

Une fois $N$ fixé on peut choisir $\varepsilon$ petit pour que

$$
\frac{O(1)}{\varepsilon^{2}}(1+O(\varepsilon))>\mu\left(J_{k}\right) \text { et } e^{2 / \pi \varepsilon}>\mu\left(J_{k}\right) \text {. }
$$

D'où

$$
\begin{aligned}
q_{k}(u) & \geq \mu\left(J_{k}\right) \int_{0}^{\pi} u^{2}(r) \sin (r) d r+O(\varepsilon) \\
& \geq \mu\left(J_{k}\right)+O(\varepsilon) .
\end{aligned}
$$

Ce qui prouve la minoration cherchée et donc le théorème 4.3.

Remarque récapitulative. Nous avons montré que pour tout $k=1, \ldots, N \lambda_{1, k}^{\varepsilon}$ converge vers $\mu\left(J_{k}\right)$ pour $\varepsilon$ tendant vers 0 , et donc est proche de $\mu_{0}$. Il reste à prouver que pour $\varepsilon$ petit ce sont les $N$ premières valeurs propres de $H_{B}$. Pour cela il suffit de montrer d'une part que pour $k=1,2, \ldots, N \lambda_{2, k}^{\varepsilon}$ est assez "loin" des $\mu\left(J_{n}\right)$ et d'autre part que pour $k \notin\{1,2, \ldots, N\}$ il en est de même de $\lambda_{1, k}^{\varepsilon}$. 
4.13. Étude de $\lambda_{2, k}^{\varepsilon}$ pour $k \in\{1,2, \ldots, N\}$. En faisant exactement le même travail que précédemment, on voit que la seule différence est ce qui se passe sur l'intervalle $J_{k}$. Sur ce dernier à l'aide du principe du min-max, on peut remplacer $\mu\left(J_{k}\right)$ par $\mu_{2}\left(J_{k}\right)$ (deuxième valeur propre du problème de Dirichlet sur $J_{k}$ ) dans l'inégalité principale. En utilisant une comparaison avec la situation euclidienne (comme dans le lemme 4.9) on peut montrer que pour $N$ assez grand on a par exemple

$$
\mu_{2}\left(J_{k}\right)>3 \mu\left(J_{k}\right) .
$$

Par ailleurs $N$ étant grand, $\mu\left(J_{k}\right)$ est lui-même assez grand, de sorte que (par exemple)

$$
\mu_{2}\left(J_{k}\right)>\mu\left(J_{k}\right)+10
$$

ce qui permet de conclure

$$
\liminf _{\varepsilon \rightarrow 0} \lambda_{2, k}^{\varepsilon}>\frac{1}{2}+\mu\left(J_{k}\right)
$$

autrement dit les $\lambda_{2, k}^{\varepsilon}$ n'interfèrent pas avec les $\lambda_{1, k}^{\varepsilon}$ pour $\varepsilon$ petit et $k \in\{1, \ldots, N\}$ à condition de choisir $N$ assez grand.

4.14. Étude de $\lambda_{1, n}^{\varepsilon}$ pour $n \notin\{1,2, \ldots, N\}$. La seule difficulté réside dans le fait que sur $\left[y_{k}, x_{k}\right]$ le potentiel de $H_{n}$ peut s'annuler. En effet $\frac{n}{\sin (r)}$ peut être de l'ordre de $-e^{N / \varepsilon}$. Nous allons donc distinguer deux cas :

1) Si $n \geq-\frac{1}{2} e^{N / \varepsilon}$ alors ce phénomène ne se produit sur aucun des intervalles $\left[y_{k}, x_{k}\right]$. On peut raisonner exactement comme avant car le potentiel y est très grand.

2) Si $n \leq-\frac{1}{2} e^{N / \varepsilon}$ alors maintenant c'est sur chacun des $J_{k}$ que le potentiel de $H_{n}$ est grand. En conséquence au voisinage des $y_{k}$ et $x_{k}$ (les bornes des $J_{k}$ ) la fonction propre est très petite (on peut utiliser un lemme analogue au lemme 4.4). Donc sur $\left[y_{k}, x_{k}\right](k=1, \ldots, N)$ nous sommes en présence d'un problème comparable au problème de Dirichlet. Les choix de $y_{k}$ et $x_{k}$ faits en première partie de cette section (l'intervalle $\left[y_{k}, x_{k}\right]$ est de l'ordre de $1 / 10$ de $J_{k}$ ) permettent d'affirmer que

$$
\mu\left(\left[y_{k}, x_{k}\right]\right) \geq 1+\mu\left(J_{k}\right) .
$$

3) De même sur $\left[2 \delta, x_{0}-\delta\right]$ où $\left[y_{N}+\delta, \pi-2 \delta\right]$ le potentiel de $H_{n}$ peut s'annuler, par exemple en un point $r_{0}$ tel que

$$
\frac{n}{\sin \left(r_{0}\right)}=-e^{\frac{1}{\varepsilon r_{0}}},
$$

mais alors il est clair qu'en dehors d'un voisinage de $r_{0}$ tendant vers 0 avec $\varepsilon$, le potentiel redevient grand. En dehors de ce voisinage on a une minoration immédiate, à l'intérieur on utilise encore une fois le problème de Dirichlet.

On procède alors comme précédemment.

4.15. Conclusion. Les $N$ premières valeurs propres de $H_{B_{\varepsilon}}$ sont les $\lambda_{1, n}^{\varepsilon}$ pour $n \in\{1, \ldots, N\}$ ( $\varepsilon$ petit) et convergent, lorsque $\varepsilon$ tend vers 0 , vers les $\mu\left(J_{n}\right)$ uniformément en les $y_{n}$ dans l'intervalle fixé. 


\section{Preuve Du ThÉORÈme 1}

5.1. Le cas des fibrés en droites triviaux. On utilise la même méthode que dans [CV1]. Soit $V$ un voisinage de $\left(\bar{\ell}_{1}, \ldots, \bar{\ell}_{N}\right)$ dans $\mathbb{R}^{N}$, où $\bar{\ell}_{k}$ est la longueur (euclidienne) de $\bar{J}_{k}$ défini au paragraphe 4.

On considère les applications $\Phi$ et $\Phi^{\varepsilon}$ définies comme suit dans $V$ :

$$
\Phi\left(\ell_{1}, \ldots, \ell_{N}\right)=\left(\mu\left(J_{1}\right), \ldots, \mu\left(J_{N}\right)\right)
$$

où $J_{k}=\left[x_{k}, y_{k}\right]$ est de longueur $\ell_{k}=y_{k}-x_{k}$;

$$
\Phi^{\varepsilon}\left(\ell_{1}, \ldots, \ell_{N}\right)=\left(\lambda_{1,1}^{\varepsilon}, \ldots, \lambda_{1, N}^{\varepsilon}\right) .
$$

Les fonctions $\Phi$ et $\Phi^{\varepsilon}$ sont continues et $\Phi^{\varepsilon}$ converge uniformément vers $\Phi$ lorsque $\varepsilon$ tend vers 0 . $\Phi$ est clairement un difféomorphisme local, on en déduit donc comme dans [CV1] que $\left(\mu_{0}, \ldots, \mu_{0}\right)$ est dans l'image de $\Phi^{\varepsilon}$ pour $\varepsilon$ assez petit et donc que $H_{B}^{\varepsilon}$ à une première valeur propre de multiplicité $N$. Cet argument montre aussi que toute multiplicité plus petite peut être atteinte.

5.2. Le cas des fibrés en droites non triviaux. Considérons le fibré $L \longrightarrow S^{2}$ de degré $p \in \mathbb{Z}$ c'est-à-dire défini par l'application de $S^{1}$ dans $S^{1} z \mapsto z^{p}$. On choisit une trivialisation sur $B(0,2 \delta)$ la boule de centre le pôle nord (noté 0 ) et de rayon $2 \delta$ et une trivialisation sur $S^{2} \backslash B(0,2 \delta)$. Si $f_{\varepsilon}$ est la même fonction que dans le paragraphe 4 (rappelons qu'elle s'annule sur $B(0, \delta)$ ), on définit une connexion sur le fibré par les 1-formes suivantes:

i) $\operatorname{Sur} S^{2} \backslash B(0,2 \delta)$

$$
\alpha=f^{\prime}(r) \sin (r) d \theta
$$

ii) $\operatorname{Sur} B(0,2 \delta)$

$$
\beta=\chi p d \theta+f^{\prime}(r) \sin (r) d \theta
$$

où $\chi$ est une fonction $\mathcal{C}^{\infty}$, nulle au voisinage de 0 et constante égale à 1 sur l'anneau $B(0,2 \delta) \backslash B(0, \delta)$.

La condition de changement de trivialisation est satisfaite.

Sur les ouverts de trivialisation, on décompose l'opérateur comme précédemment en somme d'opérateurs de Schrödinger.

Soit $s$ une section propre de $H_{B}, s$ est définie par deux fonctions

$$
\begin{aligned}
& s_{1}: B(0,2 \delta) \longrightarrow \mathbb{C}, \\
& s_{2}: S^{2} \backslash B(0,2 \delta) \longrightarrow \mathbb{C}
\end{aligned}
$$

qui satisfont

$$
s_{2}(x)=e^{i p \theta} s_{1}(x) .
$$

En décomposant en série de Fourier,

$$
\begin{aligned}
& s_{1}(x)=\sum_{n \in \mathbb{Z}} u_{n}(r) e^{i n \theta}, \\
& s_{2}(x)=\sum_{n \in \mathbb{Z}} v_{n}(r) e^{i n \theta},
\end{aligned}
$$


il vient $v_{n}=u_{n-p}$. Alors si $\lambda$ est la valeur propre considérée

$$
\begin{aligned}
\lambda \int_{S^{2}}|s|^{2} & =\int_{S^{2}}|\nabla s|^{2} \geq \int_{S^{2} \backslash B(0, \delta)}|\nabla s|^{2} \\
& \geq \int_{\delta}^{\pi}\left[\left(u_{n-p}^{\prime}(r)\right)^{2}+\left(f^{\prime}(r)+\frac{n}{\sin r}\right)^{2} u_{n-p}(r)\right] d r \\
& =q_{n}^{\delta}\left(u_{n-p}\right) .
\end{aligned}
$$

Lorsque $\varepsilon$ tend vers zéro $\lambda$ est borné, et si la section est normalisée on voit que $q_{n}^{\delta}\left(u_{n-p}\right)$ doit être borné. L'argument du lemme 4.5 montre qu'il existe $c_{0} \in[\delta, 2 \delta[$ tel que

$$
\left|v_{n}\left(c_{0}\right)\right|=\left|u_{n-p}\left(c_{0}\right)\right| \leq \varepsilon .
$$

La fonction $v_{n}$ est fonction propre de $H_{n}$ sur $B\left(0, c_{0}\right)$ (la valeur propre est $\lambda$ ) et elle satisfait presque les conditions de Dirichlet. Alors comme dans 4.5, $\int_{B\left(0, c_{0}\right)}|\nabla s|^{2}$ est grande et donc n'influence pas le comportement des premières valeurs propres de $H_{B}^{\varepsilon}$. Le reste se traite comme précédemment.

\section{RÉFÉRENCES}

[BE] P. Bérard, From vanishing theorems to estimating theorems: the Bochner technique revisited, Bull of the AMS 19, no. 2 (1988), 371-406. MR 89i:58152

[C-T] Y. Colin de Verdière, N. Torki, Opérateur de Schrödinger avec champ magnétique - Séminaire de théorie spectrale et géométrie, Grenoble, 1992-1993.

[CV1] Y. Colin de Verdière, Sur la multiplicité de la première valeur propre non nulle du laplacien, Comment. Math. Helvetici 61 (1986), 254-270. MR 88b:58140

[CV2] Y. Colin de Verdière, Le spectre du Laplacien: Surrol partiel depuis le BGM et problèmes, Séminaires et congrés S.M.F $n^{o} 1$ (ed A. L. Besse), 1996, pp. 233-252.

[GA] S. Gallot, Inégalités isopérimétriques, courbure de Ricci et invariants géométriques II, vol. 296, série I, C.R. Acad. Sc., 1983, pp. 365-368. MR 85c:53069b

[KO] B. Kostant, Quantization and unitary representations, Lectures Notes in Math., vol. 170, Springer Verlag, 1970, pp. 87-208. MR 45:3638

[TI] N. Torki, Stabilité des valeurs propres et champ magnétique sur une variété riemannienne et sur un graphe, Thèse, Univ. Grenoble, 1989.

Institut Fourier - C.N.R.S., U.R.A. 188, B.P. 74, 38402 Saint Martin D'Hères Cedex, FRANCE

Université de Savoie, Département de Mathématiques, Bât. le Chablais, 73376 Le Bourget du Lac Cedex, France

École Polytechnique - C.N.R.S., U.R.A. 169, Centre de Mathématiques, 91128 Palaiseau Cedex, France 